\title{
The Probability of Near MidAir Collisions using Level-Crossings
}

Per-Johan Nordlund, Fredrik Gustafsson

Division of Automatic Control

E-mail: perno@isy.liu.se, fredrik@isy.liu.se

5th October 2007

Report no.: LiTH-ISY-R-2830

Submitted to International Conference on Acoustics, Speech and Signal Processing 2008 (ICASSP08)

Address:

Department of Electrical Engineering

Linköpings universitet

SE-581 83 Linköping, Sweden

WWW: http://wwW. control.isy.liu.se

AUTOMATIC CONTROL

REGLERTEKNIK LINKÖPINGS UNIVERSITET

Technical reports from the Automatic Control group in Linköping are available from http://www. control.isy.liu.se/publications. 


\begin{abstract}
We consider probabilistic methods to compute the near midair collision risk using state estimate and covariance from a target tracking filter based on angle-only sensors such as digital video cameras. Existing work is only concerned with risk estimation at a certain time instant, while the focus here is to compute the integrated risk over the critical time horizon. This novel formulation leads to evaluating the probability for level-crossing. The analytic expression for this involves a multi-dimensional integral which is hardly tractable in practice. Further, a huge number of Monte Carlo simulations would be needed to get sufficient reliability for the small risks that the applications require. Instead, we propose a sound numerical approximation that leads to a one-dimensional integral which is suitable for real-time implementations.
\end{abstract}

Keywords: Near midair collision, collision avoidance, UAV, target tracking, level-crossings. 


\section{THE PROBABILITY OF NEAR MIDAIR COLLISIONS USING LEVEL-CROSSINGS}

\author{
Per-Johan Nordlund*
}

\author{
Saab Aerosystems \\ Department of Decision Support \\ 58188 Linköping \\ per-johan.nordlund@saab.se
}

\author{
Fredrik Gustafsson \\ Linköping University \\ Department of Automatic Control \\ 58183 Linköping \\ fredrik@isy.liu.se
}

\begin{abstract}
We consider probabilistic methods to compute the near midair collision risk using state estimate and covariance from a target tracking filter based on angle-only sensors such as digital video cameras. Existing work is only concerned with risk estimation at a certain time instant, while the focus here is to compute the integrated risk over the critical time horizon. This novel formulation leads to evaluating the probability for level-crossing. The analytic expression for this involves a multi-dimensional integral which is hardly tractable in practice. Further, a huge number of Monte Carlo simulations would be needed to get sufficient reliability for the small risks that the applications require. Instead, we propose a sound numerical approximation that leads to a one-dimensional integral which is suitable for real-time implementations.
\end{abstract}

Index Terms - Near midair collision, collision avoidance, UAV, target tracking, level-crossings.

\section{INTRODUCTION}

Manned aircraft flying in controlled airspace maintain a safe distance between each other using the service provided by an Air Traffic Control (ATC). ATC informs and orders human pilots to perform maneuvers in order to avoid Near MidAir Collisions (NMAC). A NMAC between two aircraft occurs if the relative distance between the two aircraft becomes less than a predefined distance. The last decade semi-automatic systems like ACAS (Airborne Collision Avoidance System) have been implemented that essentially move this responsibility from ATC to the pilot. The ACAS system, however, assumes that both aircraft exchange data on speed, height and bearing over a data link and that both systems cooperate. When operating small UAVs this assumption is often no longer valid. A typical UAV operates on altitudes where small intruding aircraft are often present that do not carry transponders.

This paper describes a method for detecting hazardous situations based on data from a passive angle-only sensor. A challenge with angle-only measuring sensors is how to deal with the significant uncertainty obtained in estimated relative distance and speed. One approach to increase accuracy in the distance estimate is to perform own platform maneuvers [1]. The method in this paper does not rely on accurate distance estimates. The reason is that the method is based on computing the probability of NMAC over a period of time. The method is robust to large uncertainties, as opposed to a method based on instantaneous probability of NMAC [2] where large uncertainties tend to diminish the resulting probability.
We present an approximate solution to the in general computationally intractable problem of computing the probability of NMAC. Although Monte-Carlo methods are known to be able to approximate probabilities arbitrarily well [3], they are also known to be computer intensive particularly when the underlying probabilities are small. Here we do not rely on Monte-Carlo methods, but instead we make use of theory for stochastic processes and level-crossings. The event corresponding to NMAC can be seen as a crossing of the safety zone boundary. By appropriate approximations of the safety zone the probability of crossing the boundary becomes computationally tractable. The same approach was applied in [2] but for the case of known initial position and velocity. Here we consider the situation with large initial uncertainties, typically as a result of tracking intruders based on angle-only sensors.

\section{PROBLEM FORMULATION}

The probability of near-midair collision (NMAC) between two aerial vehicles for a given time period $(0, T)$ is defined as

$$
\mathrm{P}\left(\operatorname{NMAC}_{(0, T)}\right)=\mathrm{P}\left(\min _{0<t<T}|s(t)|<R \cap|s(0)|>R\right),
$$

where $s(t)$ represents the relative position between the two vehicles at time $t \geq 0$ and $t$ is the prediction time. $R$ is the radius of a safety zone, which we assume has the shape of a sphere, and $R=150 \mathrm{~m}$. The definition according to (1) means that if the relative distance

$$
|s(t)|=\sqrt{s_{x}^{2}(t)+s_{y}^{2}(t)+s_{z}^{2}(t)}
$$

for any $0<t<T$ falls below $R$, no matter for how long, we have a NMAC. We are only interested in a potential NMAC in the future, thereby the added condition $|s(0)|>R$. The existing ACAS system is capable of avoiding a NMAC, given a collision scenario, with a probability which is approximately 0.95 . Therefore it is reasonable to provide a method which computes probability of collision accurately especially in the range $0.01-0.1$.

Typically, an estimate of relative position is provided by an angleonly tracking filter [4]. Target tracking will not be pursued here in detail, we simply state that based on measurements from an angle measurement unit e.g. an electro-optical sensor, the tracking filter estimates three-dimensional relative position $s(0)$ and velocity $v(0)$ in cartesian coordinates together with their covariances. To simplify the problem formulation we assume the angle measurement unit is accurate and the coordinate system is rotated such that the $x$ - axis is aligned with line of sight. This means that

$$
s_{y}(0) \equiv s_{z}(0) \equiv 0
$$

\footnotetext{
*Thanks to Saab Aerosystems and the NFFP program for funding.
} 
and the estimated state vector used for the probability computations

$$
\hat{x}(0)=\left[\begin{array}{llll}
\hat{s}_{x}(0) & \hat{v}_{x}(0) & \hat{v}_{y}(0) & \hat{v}_{z}(0)
\end{array}\right]^{T},
$$

together with its covariance matrix, noting from (2) that $\sigma_{s y}=$ $\sigma_{s z}=0$,

$$
P(0)=\left[\begin{array}{cccc}
\sigma_{s x}^{2} & \rho \sigma_{s x} \sigma_{v x} & 0 & 0 \\
\rho \sigma_{s x} \sigma_{v x} & \sigma_{v x}^{2} & 0 & 0 \\
0 & 0 & \sigma_{v y}^{2} & 0 \\
0 & 0 & 0 & \sigma_{v z}^{2}
\end{array}\right] .
$$

Without loss of generality we have assumed cross-correlation only between $s_{x}(0)$ and $v_{x}(0)$. In general this is not true, but through unitary transformations it is straightforward to obtain a $P(0)$ according to (4). We assume the tracking filter output is normally distributed, i.e.

$$
x(0) \sim \mathcal{N}(\hat{x}(0), P(0)),
$$

Note that we will only deal with a relative time scale, represented by $t=0$ as the current time on an absolute time scale. At each new time instant on the absolute time scale the tracking filter provides updated estimates of $x(0)$ and $P(0)$.

To be able to compute $\mathrm{P}\left(\operatorname{NMAC}_{(0, T)}\right)$ in (1) we need a motion model which describes how the relative position propagates over time. Here we assume the trajectory is a straight path given by

$$
\dot{s}_{i}(t)=v_{i}(t), \quad \dot{v}_{i}(t)=0 \quad \text { for } \quad i=x, y, z,
$$

i.e. only the initial conditions influence the probability of NMAC.

\section{CROSSING OF THE SAFETY ZONE}

Under the assumption that the path is straight, a geometric interpretation of a NMAC is given by Figure 1. The collision scenario is here projected such that the $\perp-$ axis is given by the direction of the vector $\left[\begin{array}{lll}0 & v_{y}(0) & v_{z}(0)\end{array}\right]^{T}$.

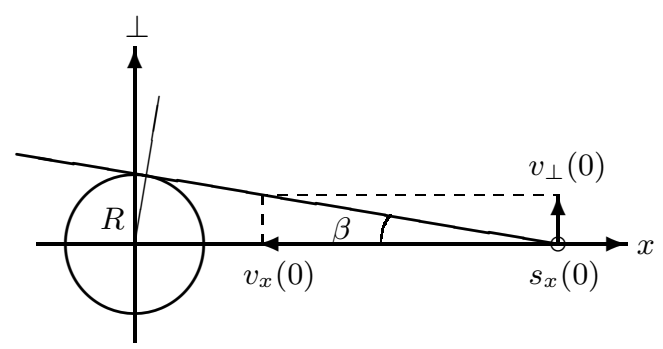

Fig. 1. Exact geometry for the limit of NMAC as seen when projected onto the plane spanned by $v_{\perp}(0)=\sqrt{v_{y}^{2}(0)+v_{z}^{2}(0)}$ and $v_{x}(0)$.

It is straightforward to show, ignoring $t=0$ for notational convenience, that

$$
\mathrm{P}\left(\operatorname{NMAC}_{(0, T)}\right)=\mathrm{P}\left(C_{1} \cap C_{2} \cap C_{3}\right),
$$

where $\cap$ denotes logical 'and', and

$$
\begin{aligned}
& C_{1}=s_{x} \sin \beta<R, \\
& C_{2}=\frac{s_{x} \cos \beta-\sqrt{R^{2}-s_{x}^{2} \sin ^{2} \beta}}{\left(v_{x}^{2}+v_{\perp}^{2}\right)^{1 / 2}}<T, \\
& C_{3}=s_{x}>R \cap v_{x}<0 .
\end{aligned}
$$

$C_{1}$ in (7) and (8) is a condition on the direction of the relative velocity, where $\beta=\arctan \frac{v_{\perp}}{\left|v_{x}\right|} . C_{2}$ is a condition on the magnitude of the relative velocity. $C_{3}$ is needed because otherwise if $s_{x}(0)<R$ it is already too late or if $v_{x}(0)>0$ there will never be a NMAC.

\section{MONTE-CARLO APPROXIMATION}

The probability according to (7) is in general very difficult to compute. A straightforward approximative solution is to use a MonteCarlo method, i.e. to draw $N$ samples of $x(0)$ from (5) and approximate the probability with the outcome of the sampling. Denote the true value of the sought probability with $p$. The set of samples is binomially distributed, $\operatorname{Bin}(N, p)$, but for a large enough $\mathrm{N}$, usually $N p(1-p)>20$ is adequate, the probability is approximated well by [5]

$$
\frac{1}{N} \sum_{i=1}^{N} C_{1}^{(i)} \cap C_{2}^{(i)} \cap C_{3}^{(i)} \sim \mathcal{N}\left(p, \sigma^{2}\right), \quad \sigma^{2}=\frac{p(1-p)}{N} .
$$

For a relative mean square error $\epsilon \leq \frac{\sigma}{p}$ we can write needed number of samples according to

$$
N \geq \frac{1-p}{\epsilon^{2} p} \approx \frac{1}{\epsilon^{2} p},
$$

where the last approximation is valid for small $p$. Assume $p=0.01$ and $3 \epsilon \leq 0.1$, i.e a relative error smaller than $10 \%$ with probability 0.997 . These values plugged into (10) suggests that we must use $N \geq 90000$. For many on-line applications this means a too high computational load.

\section{SOLUTION BASED ON APPROXIMATION OF GEOMETRY}

\subsection{Geometric Approximation}

A good approximation for a NMAC to occur is to say the relative position must cross a plane surface instead of a curved surface. Here, the plane surface is given by a circle orthogonal to line of sight and with radius $R$, see Figure 2 .

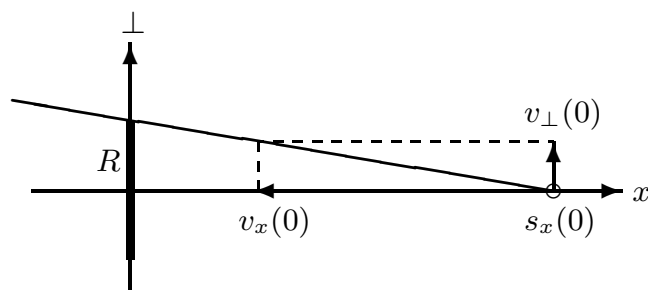

Fig. 2. Approximate geometry for the limit of NMAC as seen when projected onto the plane spanned by $v_{\perp}(0)=\sqrt{v_{y}^{2}(0)+v_{z}^{2}(0)}$ and $v_{x}(0)$.

An approximate probability of NMAC then becomes

$$
\hat{\mathrm{P}}\left(\operatorname{NMAC}_{(0, T)}\right)=\mathrm{P}\left(C_{1}^{\prime} \cap C_{2}^{\prime} \cap C_{3}^{\prime}\right),
$$

where

$$
\begin{aligned}
& C_{1}^{\prime}=s_{x} \tan \beta<R, \\
& C_{2}^{\prime}=\frac{s_{x}}{\left|v_{x}\right|}<T, \\
& C_{3}^{\prime}=s_{x}>0 \cap v_{x}<0 .
\end{aligned}
$$


Define a random variable $\tau$ according to

$$
\tau= \begin{cases}\frac{s_{x}}{\left|v_{x}\right|} & \text { if } C_{3}^{\prime} \text { is true } \\ \infty & \text { otherwise }\end{cases}
$$

where $\tau$ represents the time it takes for the distance between the two objects along line of sight to decrease to 0 . The distribution for $\tau$ is given by Lemma 1 .

Lemma 1 (Probability distribution for $\tau$ ) For a stochastic process $\left\{s_{x}(t), t \in \mathbb{R}\right\}$ with assumption (6) the probability of a down-crossing within $T$ sec is given by

$$
\mathrm{P}(\tau<T)=\int_{0}^{\infty} \int_{-\infty}^{-\frac{s}{T}} p_{s_{x}, v_{x}}(s, v) d v d s,
$$

where $p_{s_{x}, v_{x}}(s, v)$ is the joint probability function for $s_{x}$ and $v_{x}$. Proof: See Appendix A.

Now we can formulate the approximate probability of $\operatorname{NMAC}_{(0, T)}$ in (11) according to

$$
\hat{\mathrm{P}}\left(\operatorname{NMAC}_{(0, T)}\right)=\mathrm{P}\left(\tau v_{\perp}<R \cap \tau<T\right),
$$

i.e. given $\tau=t$, if $v_{\perp}$ is not large enough for the distance perpendicular to line of sight to become at least $R$ after $t$ seconds there will be a NMAC. The probability according to (15) is given by Lemma 2 .

Lemma 2 (Probability of down-crossing of a given circle) For a stochastic process $\left\{s(t)=\left[\begin{array}{lll}s_{x}(t) & s_{y}(t) & s_{z}(t)\end{array}\right]^{T}, t \in \mathbb{R}\right\}$ with assumptions (2), (4) and (6) the probability of a down-crossing within $T$ sec of a circle with $x$-axis as its normal and radius $R$ is given by

$$
\begin{aligned}
& \hat{\mathrm{P}}\left(\operatorname{NMAC}_{(0, T)}\right)=\mathrm{P}\left(\tau v_{\perp}<R \cap \tau<T\right)= \\
& \mathrm{P}(\tau<T)-\int_{\frac{R^{2}}{T^{2}}}^{\infty} p_{v_{\perp}^{2}}(v)\left(\mathrm{P}(\tau<T)-\mathrm{P}\left(\tau<\frac{R}{\sqrt{v}}\right)\right) d v,
\end{aligned}
$$

where $\mathrm{P}(\tau<T)$ is given by Lemma 1 .

Proof: See Appendix $B$.

Assuming the involved random variables are normally distributed the approximate probability for NMAC is given by (16) with the corresponding expressions for $p_{v_{\perp}^{2}}(v)$ and $\mathrm{P}(\tau<T)$ inserted. The random variable $v_{\perp}^{2}$ is a weighted sum of two non-central $\chi^{2}-$ distributed variables, i.e. with $\lambda_{i}=\frac{\hat{v}_{i}^{2}}{\sigma_{v i}^{2}}$ for $i=y$ and $z$

$$
\begin{aligned}
& p_{v_{\perp}^{2}}(v)=\sigma_{v y}^{2} \sigma_{v z}^{2} \int_{0}^{v} p_{\chi_{y}^{2}}(\xi) p_{\chi_{z}^{2}}(v-\xi) d \xi \\
& p_{\chi_{i}^{2}}(\xi)=\frac{e^{-\frac{\xi+\lambda_{i}}{2}}}{(2 \xi)^{\frac{1}{2}}} \sum_{k=0}^{\infty} \frac{\left(\frac{\lambda_{i} \xi}{4}\right)^{k}}{k ! \Gamma\left(k+\frac{1}{2}\right)} \text { for } i=y \text { and } z .
\end{aligned}
$$

The distribution $\mathrm{P}(\tau<T)$ is given by

$$
\mathrm{P}(\tau<T)=\frac{1}{2 \pi\left(1-\eta^{2}\right)^{\frac{1}{2}}} \int_{k}^{\infty} \int_{h}^{\infty} e^{-\frac{u^{2}-2 \eta u v+v^{2}}{2\left(1-\eta^{2}\right)}} d v d u
$$

where $k=-\frac{\hat{s}_{x}}{\sigma_{s x}}$ and

$$
\begin{aligned}
& h=\frac{\hat{s}_{x}+\hat{v}_{x} T}{\sqrt{\sigma_{v x}^{2} T^{2}+2 \rho \sigma_{s x} \sigma_{v x} T+\sigma_{s x}^{2}}}, \\
& \eta=-\frac{\rho \sigma_{v x} T+\sigma_{s x}}{\sqrt{\sigma_{v x}^{2} T^{2}+2 \rho \sigma_{s x} \sigma_{v x} T+\sigma_{s x}^{2}}} .
\end{aligned}
$$

\subsection{Numerical Approximation}

A simple and effective way of evaluating the outer integral in (16) is to apply Simpson's rule, i.e.

$$
\begin{aligned}
& \int_{v^{(0)}}^{v^{(2 M)}} f(v) d v=\frac{h}{3}\left(f\left(v^{(0)}\right)+4 \sum_{i=1}^{M} f\left(v^{(2 i-1)}\right)\right. \\
& \left.+2 \sum_{i=1}^{M-1} f\left(v^{(2 i)}\right)+f\left(v^{(2 M)}\right)\right)+R_{M}
\end{aligned}
$$
where $h=\frac{v^{(2 M)}-v^{(0)}}{2 M}, v^{(i)}=v^{(0)}+i h$ and $R_{M}<\frac{M h^{5}}{90}\left|f^{(4)}(\xi)\right|$
for $v^{(0)} \leq \xi \leq v^{(2 M)}$

To compute a one-dimensional normal distribution $\Phi(\cdot)$ a very accurate result is given by [6]

$$
\begin{aligned}
& \Phi(a)=\int_{-\infty}^{a} \phi(x) d x=\frac{1}{\sqrt{2 \pi}} \int_{-\infty}^{a} e^{-\frac{x^{2}}{2}} d x \approx \\
& \sqrt{\frac{1}{4}-\frac{7 e^{-\frac{a^{2}}{2}}+16 e^{a^{2}(\sqrt{2}-2)}+\left(7+\frac{\pi a^{2}}{4}\right) e^{-a^{2}}}{120}}+\frac{1}{2},
\end{aligned}
$$

for $a \geq 0$. According to [6] the relative error in (21) is less than $3 \times 10^{-4}$. This is used in order to compute an approximation of (18) according to [7]. The probability from (18), with $k, h$ and $\eta$ taken from (19), is written according to

$$
\mathrm{P}(\tau<T)=\Phi(-h)-\Phi(k) \mathrm{E}\left[\Phi\left(\frac{\eta u-h}{\sqrt{1-\eta^{2}}}\right) \mid u<k\right] .
$$

The approximation consists of replacing $u$ under the expectation in (22) with its conditional expectation $\mathrm{E}[u \mid u<k]=-\frac{\phi(k)}{\Phi(k)}$. This means that (22) is approximated with

$$
\hat{\mathrm{P}}(\tau<T)=\Phi(-h)-\Phi(k) \Phi\left(-\frac{\eta \frac{\phi(k)}{\Phi(k)}+h}{\sqrt{1-\eta^{2}}}\right) .
$$

From [8] we know we can approximate the density in (17) with a single central $\chi^{2}$ according to

$$
\hat{p}_{v_{\perp}^{2}}(v)=\frac{\sqrt{2 f}}{\sigma\left(v_{\perp}^{2}\right)} p_{\chi^{2}}\left(\frac{v-m\left(v_{\perp}^{2}\right)}{\sigma\left(v_{\perp}^{2}\right)} \sqrt{2 f}+f\right),
$$

where

$$
\begin{aligned}
p_{\chi^{2}}(\xi) & =\frac{1}{2^{\frac{f}{2}} \Gamma\left(\frac{f}{2}\right)} \xi^{\frac{f}{2}-1} e^{-\frac{\xi}{2}}, \\
m\left(v_{\perp}^{2}\right) & =\sum_{i=y}^{z} \sigma_{v i}^{2}+\hat{v}_{i}^{2}, \\
\sigma\left(v_{\perp}^{2}\right) & \left.=\left(2 \sum_{i=y}^{z} \sigma_{v i}^{4}+2 \hat{v}_{i}^{2} \sigma_{v i}^{2}\right)\right)^{1 / 2}, \\
f & =\frac{\left(\sigma\left(v_{\perp}^{2}\right)\right)^{6}}{8\left(\sum_{i=y}^{z} \sigma_{v i}^{6}+3 \hat{v}_{i}^{2} \sigma_{v i}^{4}\right)^{2}} .
\end{aligned}
$$

To summarize, the expression to numerically compute $\hat{\mathrm{P}}\left(\operatorname{NMAC}_{(0, T)}\right)$ in $(16)$ is

$$
\begin{aligned}
& \hat{\mathrm{P}}\left(\operatorname{NMAC}_{(0, T)}\right) \approx \hat{\mathrm{P}}(\tau<T)- \\
& \int_{\frac{R^{2}}{T^{2}}}^{\infty} \hat{p}_{v_{\perp}^{2}}(v)\left(\hat{\mathrm{P}}(\tau<T)-\hat{\mathrm{P}}\left(\tau<\frac{R}{\sqrt{v}}\right)\right) d v,
\end{aligned}
$$


where $\hat{\mathrm{P}}(\tau<T)$ is given by (23) and $\hat{p}_{v^{2}}(v)$ by (24). The integral in (26) is solved by applying Simpson's rule according to (20) with $v^{(2 M)}=m\left(v_{\perp}^{2}\right)+6 \sigma\left(v_{\perp}^{2}\right)$.

\section{SIMULATION RESULTS}

Figure 3 shows $\mathrm{P}\left(\mathrm{NMAC}_{(0,50)}\right)$ computed according to (26) with $M=50$ as a function of $\beta=\arctan \frac{\hat{v}_{y}}{\hat{v}_{x}}$ compared to the MonteCarlo solution from (9) when $\hat{s}_{x}=2000, \hat{v}_{x}=120, \sigma_{s x}=400$, $\sigma_{v x}=30, \rho=0.8, \hat{v}_{y}=\hat{v}_{x} \tan \beta, \hat{v}_{z}=0, \sigma_{v y}=\left(\hat{v}_{y}^{2} \sigma_{s x}^{2} / \hat{s}_{x}^{2}+\right.$ $\left.\hat{s}_{x}^{2} 10^{-6}\right)^{1 / 2}$ and $\sigma_{v z}=\hat{s}_{x} 10^{-3}$.
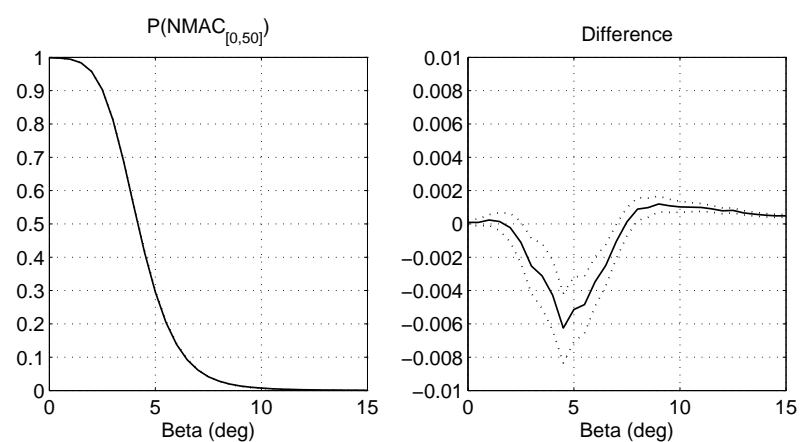

Fig. 3. The left plot shows $\mathrm{P}\left(\mathrm{NMAC}_{(0,50)}\right)$ according to (26) using $M=50$ (solid line) and the Monte Carlo solution given by (9) $(\approx 0.01$ at $\beta=9.5)$ using 500000 samples (dashed line). The right plot shows the difference between the two solutions (solid line) including the 3- $\sigma$ confidence interval for the Monte Carlo solution (dotted lines).

Table 1 shows the relative error for $\mathrm{P}\left(\operatorname{NMAC}_{(0,50)}\right)$ given by (26) evaluated at $p=0.01$ when $\sigma_{s x}$ and $\sigma_{v x}$ vary. If the requirement on the relative error is less than 0.1 at $p=0.01$ we deduce from Table 1 that if $\sigma_{s x}<400$, i.e. $\frac{\sigma_{s x}}{\widehat{s}_{x}}<0.2$, the requirement is met. The reason for the worse accuracy for larger variances is primarily due to the approximation according to (24). For better result in cases with large variances a better approximation is needed.

Table 1. Relative error $\epsilon$ at $p=0.01$ for the algorithm given by (26).

$\begin{array}{lccccccc}\sigma_{s x} & 666 & 500 & 500 & 400 & 400 & 333 & 333 \\ \sigma_{v x} & 40 & 40 & 30 & 30 & 24 & 24 & 20 \\ \epsilon & 0.5 & 0.22 & 0.22 & 0.12 & 0.12 & 0.06 & 0.07\end{array}$

\section{CONCLUSIONS}

In this paper we have presented a method to compute the probability of near midair collision between two vehicles flying along straight trajectories. Near midair collision is defined as the event of the relative position crossing the safety zone boundary. By appropriate geometric and numerical approximations the probability of crossing the boundary becomes computationally tractable. Through simulations we have shown that for a certain collision scenario the method meets the given accuracy requirement as long as the variance of estimated relative position is not too large.

\section{A. PROOF OF LEMMA 1}

Due to $v(t)=v(0)$, for a down-crossing to occur we must have $s_{x}(0)>0$ and $v_{x}(0)<0$. For a down-crossing to occur within the time frame $0<t<T$ the velocity needs to be

$$
-\infty \leq v_{x}(0) \leq-\frac{s_{x}(0)}{T}
$$

The probability for this to happen is

$$
\mathrm{P}(\tau<T)=\int_{0}^{\infty} \int_{-\infty}^{-\frac{s}{T}} p_{s_{x}, v_{x}}(s, v) d v d s
$$

\section{B. PROOF OF LEMMA 2}

Using the density for the mutually independent $v_{\perp}$ and $\tau$ we have

$$
\begin{aligned}
\mathrm{P}\left(\tau v_{\perp}<R \cap \tau<T\right) & =\mathrm{P}\left(v_{\perp}^{2}<\frac{R^{2}}{\tau^{2}} \cap \tau<T\right) \\
& =\int_{0}^{T} \int_{0}^{\frac{R^{2}}{t^{2}}} p_{v_{\perp}^{2}}(v) p_{\tau}(t) d v d t .
\end{aligned}
$$

Changing the order of computation in (29) yields

$$
\begin{aligned}
& \mathrm{P}\left(\tau v_{\perp}<R \cap \tau<T\right)= \\
& \int_{\frac{R^{2}}{T^{2}}}^{\infty} \int_{0}^{\frac{R}{\sqrt{v}}} p_{v_{\perp}^{2}}(v) p_{\tau}(t) d t d v+\int_{0}^{\frac{R^{2}}{T^{2}}} \int_{0}^{T} p_{v_{\perp}^{2}}(v) p_{\tau}(t) d t d v= \\
& \mathrm{P}(\tau<T)-\int_{\frac{R^{2}}{T^{2}}}^{\infty} p_{v_{\perp}^{2}}(v)\left(\mathrm{P}(\tau<T)-\mathrm{P}\left(\tau<\frac{R}{\sqrt{v}}\right)\right) d v
\end{aligned}
$$

\section{REFERENCES}

[1] O. Shakernia, W-Z. Chen, and V. M. Raska, "Passive ranging for UAV sense and avoid applications," in Proceedings of Infotech@Aerospace, 2005.

[2] M. Prandini, J Hu, J Lygeros, and S. Sastry, "A probabilistic approach to aircraft conflict detection," IEEE Transactions on Intelligent Transportation Systems, vol. 1, no. 4, pp. 199-220, 2000.

[3] L. Yang, J. H. Yang, J. Kuchar, and E. Feron, "A real-time Monte Carlo implementation for computing probability of conflict," in Proceedings of the AIAA Guidance, Navigation and Control Conference and Exhibit, 2004.

[4] S. Blackman and R. Popoli, Design and Analysis of Modern Tracking Systems, Artech House, 1999.

[5] A. Gut, An Intermediate Course in Probability, Springer-Verlag, 1995.

[6] R.J. Bagby, "Calculating normal probabilities," The American Mathematical Monthly, vol. 102, no. 1, pp. 46-49, Jan 1995.

[7] D.R. Cox and N. Wermuth, "A simple approximation for bivariate and trivariate normal integrals," International Statistical Review, vol. 59, no. 2, pp. 263-269, 1991.

[8] J.P. Imhof, "Computing the distribution of quadratic forms in normal variables," Biometrika, vol. 48, no. 3/4, pp. 419-426, 1961. 